\title{
Multi-agent Approach to the Two-Second Driving Rule
}

\author{
Diego Uribe*, Enrique Cuan \\ Instituto Tecnológico de la Laguna, Blvd. Revolution and Cuauhtémoc, Torreón, Coah., México. \\ * Corresponding author. Email: diegouribe@acm.org \\ Manuscript submitted January 5, 2017; accepted April 7, 2017. \\ doi: $10.17706 /$ jcp.13.2.168-175
}

\begin{abstract}
In this work we describe the development of a computational model based on agents to simulate the two-second driving rule. In other words, we are interested in determining the traffic conditions under which the rule appears to be safe as well as those traffic conditions under which the rule has elements of risk. The model we develop considers the setting of parameters that have impact on the stopping distance. As a result, the experimental simulation allows us to examine and, in some cases, verify theoretical concepts of collisions. Our approach also illustrates how agent-based modeling offers a plausible alternative in representing complex systems such as vehicular traffic.
\end{abstract}

Key words: Agent-based modeling, traffic model, micro, macro levels.

\section{Introduction}

Heavy traffic in big cities causes many urban problems. These include: wasted time, poor fuel efficiency, high pollution, stress and unfortunately, an increase in the accident rate [1]. Given this scenario, and with the aim of providing guidelines for driving safely, one of the main activities of a traffic engineer is to take care of the traffic flow and the traffic density for making decisions such as expanding the number of lanes, or installing traffic lights. For example, vehicles travelling close together are more likely to crash. So, to avoid an increase in the accident rate of a particular road, the traffic engineer analyse the traffic flow to determine a pertinent separation between vehicles.

To help avoid car accidents, a common tip for new vehicle drivers is the two-second rule. This suggests that it is good driving practice to keep the distance between two cars as being two seconds from the car immediately in front. However, even though most drivers have no clear evidence how well grounded such a rule is, drivers understand the importance of speed in helping to control their vehicles.

In this work, a computational model has been implemented to investigate the theoretical framework behind the two-second driving rule. In fact, concepts from physics around car collisions such as workenergy principle and friction are used to help understand car collisions, along with basic concepts of traffic flow such as speed, density and stopping distance. The theoretical framework of the traffic systems has been studied from various perspectives such as fluid dynamics [2] or kinetic theory of gases [3]. In our particular work, we develop a computational model rooted in complex systems.

In recent years there has been an increasing interest in techniques for dealing with the complexities of dynamic scenarios. According to the definition of a complex system, as a system characterized by an environment in which multiple independent elements interact with each other giving rise to an emerging phenomenon [4], [5], to analyse vehicular traffic from this perspective seems to be a plausible approach. In fact, taking into account the multiple factors to be considered when driving a vehicle (e,g. the presence of 
other vehicles, road conditions, traffic lights, traffic accidents, pedestrian crossing), a traffic system can also be characterized as a complex system.

In this way, since agent based modeling is a computational methodology that allows us to model complex systems [6], we analyze in this work the basic elements to be considered in vehicular traffic by using modeling based on agents. To be more specific, the vehicles are represented as agents, whereas the road is represented as the environment in which the vehicles travel and interact. Likewise, to simulate the twosecond driving rule, the micro and macro levels that characterize a complex system are also considered in our model.

In short, in this paper we investigate the essential parameters to simulate the two-second driving rule and thus, to determine the traffic conditions under which the driving rule is safety as well as those traffic conditions in which the rule is risky. In other words, by setting the parameters' values such as speed, density and time headway, we can provide an appropriated guideline for driving safely.

The theoretical framework of the methodology adopted in the development of the traffic model is described in section 3. As it is briefly mentioned lines above, the model represents the simulation of the two-second driving rule based on agents so the properties, behaviour and interactions between autos is detailed. Then, the basic parameters for an elemental runtime analysis are presented in section 4 as well as the particular parameters' values for driving safely. In the meantime, other models for describing traffic flows are briefly mentioned in the next section.

\section{Related Work}

In this section, we briefly narrate other models for describing traffic flows. Traffic flow theory is a scientific field relatively young. The first traffic problems in the past century were solved by the implementation of rule of thumb methods. However, at the beginning of the 1950s, the turning point in the field of traffic engineering came when Glen Wardrop described traffic flows by using mathematical representations [7].

The first popular traffic flow model, commonly known as the LWR model, was based on fluid dynamics, a discipline for studying fluids (liquids and gases) in motion [2], [8]. In fact, traffic flow model makes use of numerical analysis and algorithms to solve and analyze problems that involved fluid flows. The interactions of liquids and gases are used as an analogy to model the interactions among vehicles.

Another interesting work was the traffic model implemented by Prigogine and Herman who drew an analogy between gas-kinetic and the velocity distributions [3]. It is basically a stochastic model of traffic flow in which the "microgoals" of each driver are modified by the interaction with other drivers. Then, the central idea is to predict how much the "microgoals" of the driver have been modified by making use of a kinetic equation defined in terms of the desire of the driver and the interactions with other vehicles.

Statistical physics represents a more recent framework in traffic flow modelling. Particularly, the cellular automata model developed by Nagel and Schreckenberg is based on particles systems where the cells have varying states over time [9].

\section{Theoretical Framework}

The theoretical framework that supports our traffic model is described in this section. First, we briefly define the microscopic and macroscopic properties of traffic flow to study vehicular transport systems as well as the concept of stopping distance. Then, we present the essential concepts of agent-based modeling, the methodology for designing and building the basic traffic model.

\subsection{Microscopic and Macroscopic Properties of a Traffic Flow}

Broadly speaking, the drivers and vehicles, that is, the actors of a vehicular transport system denote the 
microscopic characteristics, whereas the interactions between drivers denote the macroscopic characteristics of traffic flows [10].

The microscopic properties of a traffic flow are described, first, in terms of a single vehicle, and then, by considering two consecutive vehicles. The main characteristics to describe a vehicle in a traffic lane are: length, position, velocity and acceleration.

Driving safely requires being aware of how close another car is. Thus, by considering two consecutive vehicles in a traffic lane, we have to very important properties:

- space headway: it is defined as the space gap between two consecutive vehicles: a follower and its leader (i.e. predecessor), that is measured from the follower's rear bump to its leader's rear bump. Such space gap is usually expressed in metres.

- time headway: it is defined as the time to travel the space headway at the current velocity. Such time gap is usually expressed in secs.

- The macroscopic properties of a traffic flow are considered in terms of an aggregate level of multiple vehicles in a traffic stream. We begin with density: a measure to determine how crowded a section of a road is. Density, represented by $k$ and expressed in terms of veh $/ \mathrm{m}$, is estimated by the following expression:

$$
k=\frac{n_{x}}{x}
$$

where $n_{x}$ denotes the number of vehicles in a road of length $x$.

The second macroscopic property, known as flow, is concerned with time: a temporal measure to determine the number of vehicles passing at a particular point of the road during a particular period of time. Flow is represented by $q$ and expressed in terms of veh/s, is estimated by the following expression:

$$
q=\frac{n_{t}}{t}
$$

where $n_{t}$ denotes the number of vehicles moving during a particular period of time $t$.

Average speed is the third macroscopic property and it is concerned with multiple rather than individual speeds. Average speed, represented by $u_{t}$, is estimated by the following expression:

$$
u_{t}=\frac{\sum_{i=1}^{n} u_{i}}{n}
$$

where $n$ denotes the number of vehicles, and $u_{i}$ the speed of an individual vehicle.

\subsection{Stopping Distance and Friction}

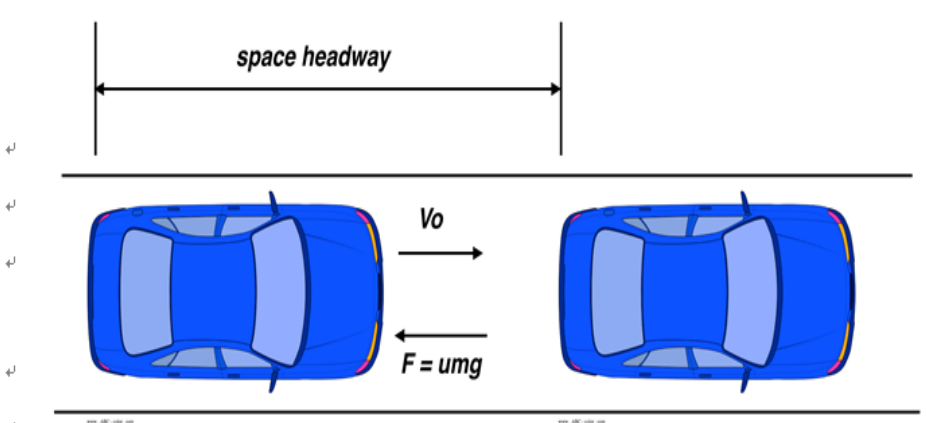

Fig. 1. Work-energy-principle and space headway. 
The elements to be considered when deceleration of the vehicle is required are multiple. Road, tires and brakes conditions are key elements to stop at a reasonable distance in order to avoid an accident. Stopping distance, the concept that represents the interaction between road, tires and brakes conditions, is fundamental to determine how long it will take a driver to stop a vehicle. Thus, when a driver makes use of the brakes, the purpose is to minimize the stopping distance.

The work-energy principle proves how to reduce the kinetic energy to zero [11]. Fig. 1 illustrates how the friction force must do enough work on the car-following to counteract its kinetic energy to zero. Then, when a driver brakes, the force of the static friction comes into operation to restrain the car-following's speed. In this way, the minimum stopping distance for a car is determined by the friction between the tires and the road. The stopping distance denoted by $d$, is estimated by the following expression:

$$
d=\frac{v_{0}^{2}}{2 u g}
$$

where $v_{0}$ denotes speed of the car, and $u$ friction.

\subsection{Agent-Based Modeling}

The approach for developing the basic traffic model is based on one of the primary methodologies to study complex problems: agent-based modeling. A complex system is defined as a system characterized by an environment in which multiple individual and independent elements interact with each other giving rise to an emerging phenomenon [4], [5]. The complexity of a system is observed in natural and social phenomena that are nondeterministic and have distributed mechanisms and control. Thus, to simulate the interactions among agents to recreate the macroscopic properties that emerge is fundamental to make sense of the phenomena to investigate.

Agent based modeling is a computational methodology that allows us to model complex systems [6]. More precisely, ABM is a computational modeling paradigm that enables us to describe a complex system in terms of agents, environment, and interactions. While agents denote the basic ontological unit of the model, the environment represents the world in which the agent lives. In this work, we make use of Agent based modeling to represent vehicular traffic flows. To be more specific, the vehicles are represented as agents, whereas the road is represented as the environment in which the vehicles travel and interact. The micro and macro levels that characterize a complex system are also analyzed to present the empirical relationships of a traffic system in terms of density, flow and speed.

\section{Two-Second Rule Model}

In this section we present the elements for developing the two-second rule model as well as the results obtained with diverse configurations of the main parameters. First, we describe the scenario in which the agents interact and then, the section concludes with the presentation of the experimental simulation to examine theoretical concepts of collisions.

\subsection{Scenario}

The two-second rule model has been developed with NetLogo [12]. NetLogo is a programming language used to create models based on agents and has also proved to be a well suited tool for modeling complex systems evolving over time. One of the most appealing features of NetLogo is the capacity to investigate the connections between the micro-level behaviour of individuals and macro-level patterns that emerge from their interactions [13].

Since velocity is an intrinsic parameter to any traffic model, an appropriated representation of distance 
and time is essential for a traffic simulation system [14]. The model depicts a traffic lane in which the velocity of the cars is determined according to the available space between them: a driver might accelerate o decelerate to the extent of the distance between his vehicle and the next. In other words, taking care of the distance is essential for the safety control of a car.

To achieve a better representation of the distance between cars we focus our attention in one of the fundamental microscopic traffic characteristics: the space headway. As we mentioned in section 3, the space headway between two consecutive vehicles: a follower and its leader (i.e. predecessor), is defined as the space gap measured from the follower's rear bump to its leader's rear bump. Such space gap is usually expressed in metres [10].

Since NetLogo is a generic tool for agent-based modeling, the configuration of the environment depends on the domain to be modeled. In fact, NetLogo represents the environment (called "world") as a bidimensional space that is divided up into a grid of "patches", where a "patch" is the basic unit used by NetLogo to represent the environment in which the agents interact. For example, in the classic Wolf-Sheep Predation model, the environment is represented by a landscape where a "patch" denotes a minute portion of the landscape, that is, a minute portion of grass. In the two-second rule model, the environment is represented by a road space where a "patch" denotes a space of $5 \mathrm{~m}$, the standard size of a vehicle.

Also, in order to perform all calculations involving distance, it is essential to define in NetLogo the maximum size of the environment in terms of a scale. In our model, the scenario is represented with a configuration of a 1: 5 scale. In this way, a road space of 500 meters is modeled with 100 patches.

\subsection{Simulation, Results and Analysis}

The model is defined in terms of five basic parameters, some of them already described in the previous section: density: to determine how crowded the road is, speed, acceleration, brake, and time headway: time required to travel the space headway at the current velocity.

According to the length of the road space (500 meters) and the standard size of a car $(5 \mathrm{~m})$, the maximum value for density is 40 . In other words, a value close to 40 denotes a crowded road. Since the average rate of acceleration of ordinary cars is between 3 and $4 \mathrm{~m} / \mathrm{s} 2$, the value of this parameter corresponds to this range. Brake denotes the rate at which the driver can safely decelerate the car. A normal value for a vehicle traveling with decent tires on a dry road is $4.5 \mathrm{mps}$.

Time headway is the key parameter of the model. Since time headway denotes the time required to travel the space headway at a particular speed, time headway is the essential parameter to simulate the twosecond driving rule. In our experimentation we try two values: 2 and 3.

Table 1 shows how when density is at its lowest value, and speed is at its highest value, there are no collisions. However, when density increase and speed is keeping at its highest value, collisions come out. Also, we notice how when speed is not so high ( $80 \mathrm{kph}$ ), we have collisions with large values of density.

Table 1. Density, Speed and Collision with Time Headway $=2$

\begin{tabular}{ccccccc}
\hline Density & Speed & Collision & Speed & Collision & Speed & Collision \\
\hline 10 & 120 & No & 100 & No & 80 & No \\
15 & 120 & Yes & 100 & Yes & 80 & No \\
20 & 120 & Yes & 100 & Yes & 80 & Yes \\
\hline
\end{tabular}

Fig. 2 shows the speed reached by a particular car during a period of 5 minutes (300 ticks where tick represents the basic unit of time provided in NetLogo). Fig. 2a, 2b and $2 \mathrm{c}$ have been obtained with a density value of 10,15 and 20 respectively. Fig. 2a (upper figure) shows how there are no collisions when density is 
at its lowest value and we travel at maximum speed. However, Fig. $2 \mathrm{~b}$ and $2 \mathrm{c}$ show how when density increases and speed is keeping at its highest value, collisions come out. The collision in Fig. $2 \mathrm{~b}$ occurred after 73 seconds whereas in Fig. 2c the collision occurred after 36 seconds.

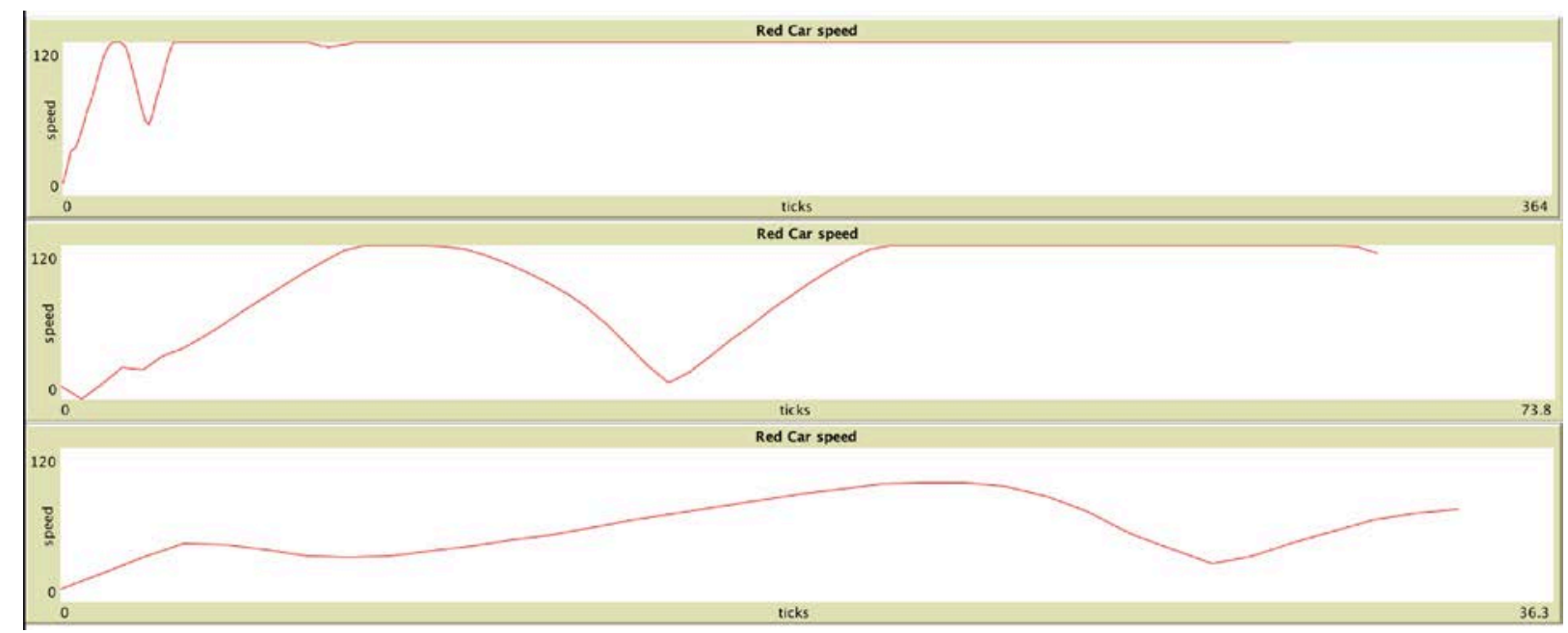

Fig. 2. Car travelling at a speed of $120 \mathrm{kps}$ whit time headway $=2$.

However, the results are different when we try a time headway of 3. Fig. 3 shows four subgraphs: Fig. 3a, $3 \mathrm{~b}, 3 \mathrm{c}$ and $3 \mathrm{~d}$ have been obtained with a density value of $10,15,25$ and 35 respectively. Two key points are to be noticed in this figure. First, there are no collisions regardless of the density's value. Second, speed gradually decreases, which denotes a more preventive driving habit. Since the time headway has been increased, the use of the brake comes into operation one second before.

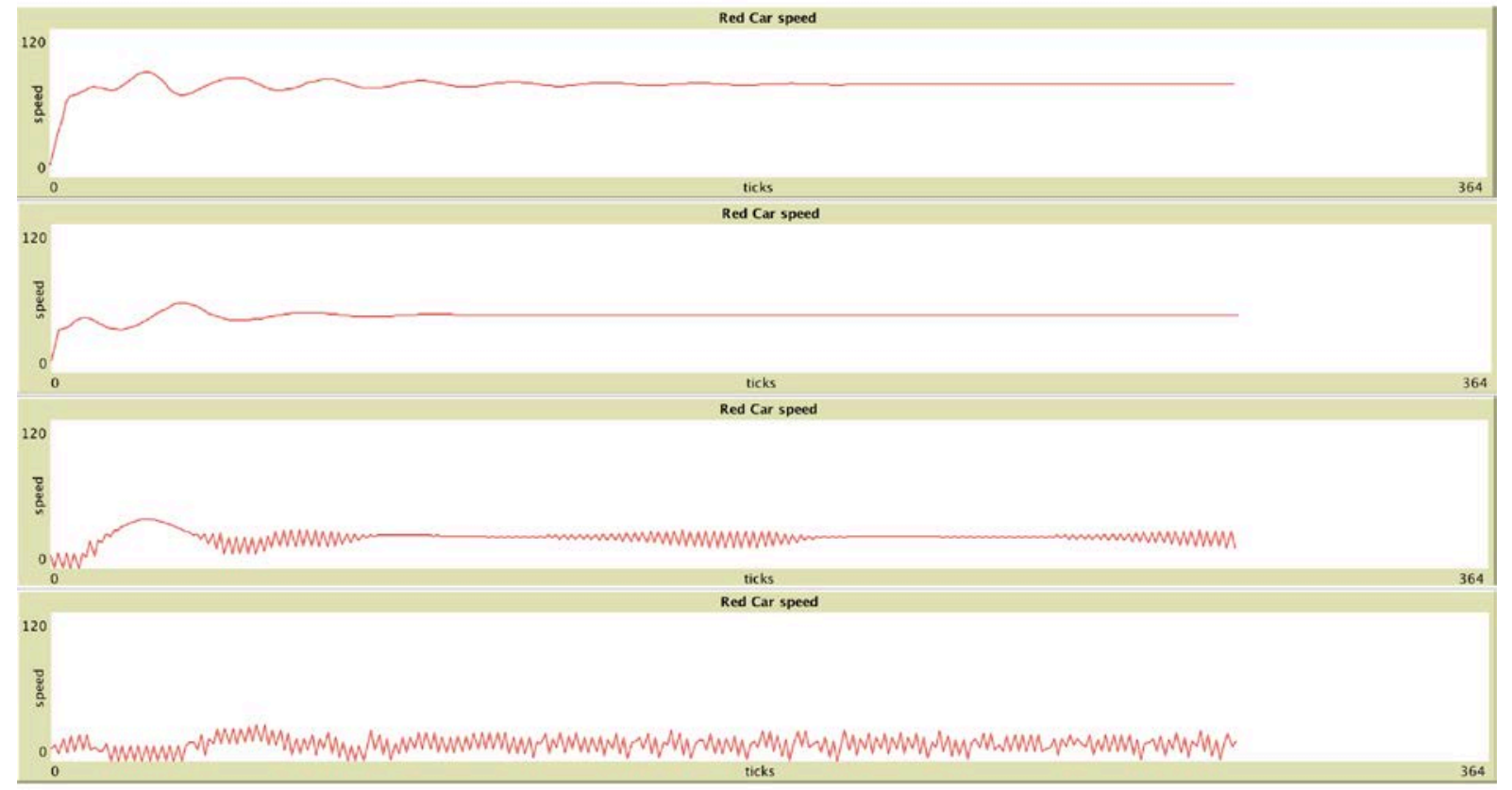

Fig. 3. Car travelling at a speed of 120 kps whit time headway $=3$.

The results of our experimentation exhibit how the two-second rule is not appropriated for traveling at high speeds. The equation 4 shows the real impact of velocity on stopping distance: the initial velocity is squared rather than doubled. This means that stopping distance increases exponentially as we drive faster 
[15]. As we said in the previous paragraph, a time headway greater than two corresponds a space headway bigger that is fundamental to decrease the speed and reduce the influence of stopping distance.

Last but not least, as fundamental part of our analysis work, we put forward the consideration of some of the shortcomings of the two-second rule model. First, the expression 4 to estimate the stopping distance is independent of vehicle's mass. In other words, the stopping distance for a large truck is the same as for a car. Then, it is interesting as part of future work to verify this case by including diverse type of vehicles in the model. Second, it would also be interesting to include in the model diverse conditions of the road such as wet roads and snow. To study the influence of different coefficients of friction between the tires and the roadway is worth of exploration. Finally, the inclusion of human factors such as reaction time is also a key element to be considered for developing a more realistic traffic simulation system.

\section{Conclusion and Future Work}

In this paper we investigate the essential parameters to simulate the two-second driving rule and to determine the traffic conditions under which the rule appears to be safe as well as those conditions under which the rule has elements of risk. The model developed for the experimental simulation allows to analyse and verify theoretical concepts of collisions. In fact, the setting of parameters such as speed, density and time headway enable us to measure the influence on the stopping distance. Moreover, the approach used to develop our model illustrates how agent-based modeling offers a plausible alternative in representing complex systems such as vehicular traffic.

About future work, many dynamic aspects of traffic flows have a huge influence over developing a more realistic traffic model. Dynamic and complex aspects to be modeled such as the conditions of the road (e.g. wet roads), human factors (e.g. reaction time) and diverse type of vehicles are undoubtedly interesting aspects to investigate and to include in the developing of a traffic simulation system.

\section{Acknowledgment}

John Arundel provided useful comments and assistance in the development of this manuscript. This research was supported by the Programa para el Desarrollo Profesional Docente (PRODEP) of Mexico.

\section{References}

[1] Greengard, S. (2015). Smart transportation networks drive gains. Communications of the ACM, 58(1), 25-27.

[2] Lighthill, M., \& Whitham, G. (1955). On kinematic waves: A theory of traffic flow on long crowded roads. Proceedings of the Royal Society: Vol. A229. (pp. 317-345).

[3] Prigogine, I., \& Herman, R. (1971). Kinetic Theory of Vehicular Traffic. New York: Elsevier.

[4] Holland, J. (1995). Hidden order: How adaptation builds complexity. Helix Books/Addison-Wesley.

[5] Kauffman, S. (1995). At Home in the Universe: The Search for the Laws of Self-Organization and Complexity. Oxford University Press.

[6] Wilensky, U., \& Rand, W. (2015). An Introduction to Agent-Based Modeling. Cambridge Massachusetts: The MIT Press.

[7] Wardrop, J. (1952). Some theoretical aspects of road traffic research. Proceedings of the Institution of Civil Engineers: Vol. 1.

[8] Richards, P.I. (1956). Shockwaves on the highway. Operations Research, 4, 42-51.

[9] Nagel, K., \& Schreckenberg, M. (1992). A cellular automaton model for freeway traffic. Journal de Physique I France, 2, 2221-2229.

[10] Maerivoet, S., \& De Moor, B. (2005). Traffic flow theory. Eprint arXiv: Physics. 
[11] Serway, R., \& Jewett, J. (2013). Physics for scientists and engineers. Cengage Learning.

[12] Wilensky, U. (1999). Center for Connected Learning and Computer-Based Modeling. Evanston: Northwestern University.

[13] Janota, A., \& Rastocny, K. (2005). Multi-agent approach to traffic simulation in netlogo environment level crossing model. Proceedings of the 5th International Conference Transport Systems Telematics TST05-2005.

[14] Mayrhofer, C. (2015). Performance, scale and time in agent-based traffic modeling with netlogo. Journal for Geographic Information Science. 567-570.

[15] Townsend, B. (2002). The physics of car collisions. Retrieved from http://ffden2.phys.uaf.edu/211_fall2002.web.dir/ben_townsend/PhysicsofCarCollisions.htm

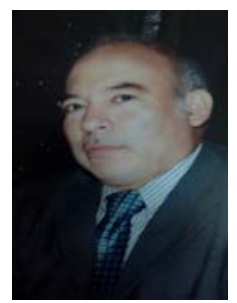

Diego Uribe was born in Torreon, Coah., Mexico. He obtained his PhD in the Department of Computer Science of the University of Sheffield, UK in 2004. His main areas of interest are natural language processing and machine learning. He works in the Instituto Tecnológico de La Laguna in Torreon, Coah., Mexico in the Postgraduate Department. He is currently working in a project about intelligent systems and agent-based modeling. 